\title{
OPTIMAL ERROR ESTIMATE FOR SEMI-DISCRETE GAUGE-UZAWA METHOD FOR THE NAVIER-STOKES EQUATIONS
}

\author{
JAE-Hong Pyo
}

\begin{abstract}
The gauge-Uzawa method which has been constructed in [11] is a projection type method to solve the evolution Navier-Stokes equations. The method overcomes many shortcomings of projection methods and displays superior numerical performance $[11,12,15,16]$. However, we have obtained only suboptimal accuracy via the energy estimate in [11]. In this paper, we study semi-discrete gauge-Uzawa method to prove optimal accuracy via energy estimate. The main key in this proof is to construct the intermediate equation which is formed to gauge-Uzawa algorithm. We will estimate velocity errors via comparing with the intermediate equation and then evaluate pressure errors via subtracting gauge-Uzawa algorithm from Navier-Stokes equations.
\end{abstract}

\section{Introduction and the gauge-Uzawa method}

Given an open bounded polygon (or polyhedron) $\Omega$ in $\mathbb{R}^{d}$ with $d=2$ (or 3 ), we consider the time dependent Navier-Stokes Equations (NSE):

$$
\begin{aligned}
\mathbf{u}_{t}+(\mathbf{u} \cdot \nabla) \mathbf{u}+\nabla p-\mu \triangle \mathbf{u} & =\mathbf{f}, & & \text { in } \Omega, \\
\nabla \cdot \mathbf{u} & =0, & & \text { in } \Omega, \\
\mathbf{u}(\mathbf{x}, 0) & =\mathbf{u}^{0}, & & \text { in } \Omega,
\end{aligned}
$$

with vanishing Dirichlet boundary condition $\mathbf{u}=\mathbf{0}$ on $\partial \Omega$ and pressure meanvalue $\int_{\Omega} p=0$. One of the main numerical difficulties in solving (1.1) is that the velocity and the pressure are coupled together through the incompressibility condition $\nabla \cdot \mathbf{u}=0$. The original projection method was introduced by Chorin [2] and Temam [17] in the late 60's to decouple the computation of velocity from the pressure, it quickly gained popularity in the computational

Received July 8, 2008.

2000 Mathematics Subject Classification. 65M12, 65M15, 76D05.

Key words and phrases. projection method, gauge method, Uzawa method, gauge-Uzawa method, Navier-Stokes equation, incompressible fluids.

This work was supported by the Korea Research Foundation Grant funded by the Korean Government (MOEHRD, Basic Research Promotion Fund) (KRF-2007-331-C00043). 
fluid dynamics community, and over the years, an enormous amount of efforts have been devoted to develop more accurate and efficient projection schemes.

Recently, E and Liu in [5] constructed a consistent projection type scheme which is called the gauge method. It introduces new variables a and $\phi$ (gauge) such that $\mathbf{u}=\mathbf{a}+\nabla \phi$ and couple them via the boundary condition $\mathbf{a}=-\nabla \phi$ for $\mathbf{u}=\mathbf{0}$. The method has been studied in [18] using asymptotic method, in [10] employing variational technique, and in [1, 15] applying normal mode analysis. The boundary coupling is responsible for accuracy degradation in problems as illustrated in [11], specially for singular solutions due to reentrant corners. It also makes the use of finite element methods problematic for space discretization. To overcome these difficulties, Nochetto and Pyo in [11] constructed the gauge-Uzawa Algorithm 1 below which inherits some beneficial properties of both the gauge method and the Uzawa method and avoids dealing with boundary derivatives.

The numerical analysis of projection type methods are usually carried out by using an energy method or a normal mode analysis: the energy method is capable of providing rigorous estimates for general settings but often overlooks particular error structures of projection errors; on the other hand, the normal mode analysis is only applicable to very special domains such as a periodic channel or a quarter plane but often reveals more precise information on the error behaviors. So the gauge-Uzawa Algorithm 1 has been studied via both normal mode analysis in $[1,15]$ and energy method in [11]. The normal mode solution of the BDF2 time discrete gauge-Uzawa has been proved fully accurate on quarter plane [1] and on periodic channel [15]. Pyo and Shen in [15] compared normal mode solutions of several projection methods and discovered that only the gauge-Uzawa has no spurious boundary layer term.

In [11], authors proved its stability and estimated errors for both velocity and pressure via energy method as summarized in (1.10)-(1.12) below. The estimates are performed very rigorously under realistic regularity requirements, but those are only suboptimal for velocity in $L^{2}\left(0, T ; \mathbf{H}^{1}(\Omega)\right)$ and for pressure in $L^{2}\left(0, T ; L^{2}(\Omega)\right)$. GU-FEM has been degraded by the sub-optimality, even though it has many advantages numerically and theoretically $[10,15,12,16]$. The purpose of this paper is to prove the optimal convergence which are summarized in Theorems 1.2 and 1.3 .

Since the gauge-Uzawa method has been precisely derived from the gauge method via changing variables in [11], we here introduce the gauge-Uzawa method briefly and directly from the backward Euler time discrete formula for linearized NSE:

$$
\frac{\mathbf{u}^{n+1}-\mathbf{u}^{n}}{\tau}+\nabla p^{n+1}-\mu \triangle \mathbf{u}^{n+1}=\mathbf{f}\left(t^{n+1}\right) .
$$

Gauge-Uzawa hires artificial variables $\widehat{\mathbf{u}}^{n+1}$ and $\rho^{n+1}$ satisfying $\widehat{\mathbf{u}}^{n+1}=\mathbf{u}^{n+1}$ $\nabla \rho^{n+1}$, and rewrite $\rho^{n+1}$ as $\rho^{n+1}=\phi^{n+1}-\phi^{n}$, which means

$$
\widehat{\mathbf{u}}^{n+1}=\mathbf{u}^{n+1}-\nabla \rho^{n+1}=\mathbf{u}^{n+1}-\nabla\left(\phi^{n+1}-\phi^{n}\right) .
$$


Inserting (1.3) into (1.2) yields

$\frac{\widehat{\mathbf{u}}^{n+1}-\mathbf{u}^{n}}{\tau}+\nabla\left(p^{n+1}+\frac{\phi^{n+1}-\phi^{n}}{\tau}-\mu \triangle\left(\phi^{n+1}-\phi^{n}\right)\right)-\mu \triangle \widehat{\mathbf{u}}^{n+1}=\mathbf{f}\left(t^{n+1}\right)$.

If we impose $\phi^{n+1}$ as a solution of the backward Euler time discrete heat equation

$$
\frac{\phi^{n+1}-\phi^{n}}{\tau}-\mu \triangle \phi^{n+1}=-p^{n+1}
$$

then we obtain a time discrete momentum equation

$$
\frac{\widehat{\mathbf{u}}^{n+1}-\mathbf{u}^{n}}{\tau}+\mu \nabla \triangle \phi^{n}-\mu \triangle \widehat{\mathbf{u}}^{n+1}=\mathbf{f}\left(t^{n+1}\right)
$$

To deal with the third order term $\nabla \triangle \phi^{n}$, which is a source of trouble due to lack of commutativity of the differential operators at the discrete level, we introduce the variable $s^{n+1}=\triangle \phi^{n+1}$ and note the connection with the Uzawa iteration:

$$
\begin{aligned}
s^{n+1} & =\triangle \phi^{n+1} \\
& =\triangle \phi^{n}-\nabla \cdot \widehat{\mathbf{u}}^{n+1}=s^{n}-\nabla \cdot \widehat{\mathbf{u}}^{n+1} .
\end{aligned}
$$

If one take divergence on each term in (1.3), then $\nabla \cdot \mathbf{u}^{n+1}=0$ gives

$$
-\triangle \rho^{n+1}=\nabla \cdot \widehat{\mathbf{u}}^{n+1} \text {. }
$$

One advantage of gauge-Uzawa method comes from the gauge formula (1.4). Since no initial and boundary conditions for pressure are given in NSE, some projection methods require an effort to find a suitable initial value or suffer from artificially imposed boundary condition on pressure [13]. In the view of (1.4), we can impose initial and boundary conditions on the non-physical variable $\phi^{n+1}$ instead of on pressure $p^{n+1}$, and the value of $p^{n+1}$ is not changed by the imposed conditions on $\phi^{n+1}$. But the Neumann condition $\partial_{\boldsymbol{\nu}} \phi^{n+1}=0$ on boundary is essential to make the compatibility condition hold on (1.6). However, there is no special restriction on initial $s^{0}$, and so we take simply $s^{0}=0$. Finally, we arrive at the gauge-Uzawa method by combining above equations after add an available time discrete convection term.

Algorithm 1 (Gauge-Uzawa method). Start with $s^{0}=0$ and $\mathbf{u}^{0}=\mathbf{u}(0, \mathbf{x})$. Repeat for $1 \leq n \leq N$,

Step 1: Find $\widehat{\mathbf{u}}^{n+1}$ as the solution of

$$
\begin{aligned}
\frac{\widehat{\mathbf{u}}^{n+1}-\mathbf{u}^{n}}{\tau}+\left(\mathbf{u}^{n} \cdot \nabla\right) \widehat{\mathbf{u}}^{n+1}+\mu \nabla s^{n}-\mu \triangle \widehat{\mathbf{u}}^{n+1} & =\mathbf{f}\left(t^{n+1}\right), \\
\widehat{\mathbf{u}}^{n+1} & =\mathbf{0}, \quad \text { in } \Omega,
\end{aligned}
$$

Step 2: Find $\rho^{n+1}$ as the solution of

$$
\begin{aligned}
-\Delta \rho^{n+1} & =\nabla \cdot \widehat{\mathbf{u}}^{n+1}, & & \text { in } \Omega, \\
\partial_{\boldsymbol{\nu}} \rho^{n+1} & =0, & & \text { on } \partial \Omega,
\end{aligned}
$$


Step 3: Update $\mathbf{u}^{n+1}$ and $s^{n+1}$

$$
\begin{aligned}
& \mathbf{u}^{n+1}=\widehat{\mathbf{u}}^{n+1}+\nabla \rho^{n+1}, \\
& s^{n+1}=s^{n}-\nabla \cdot \widehat{\mathbf{u}}^{n+1} .
\end{aligned}
$$

Remark 1.1 (Pressure). One may compute the pressure whenever necessary as

$$
p^{n+1}=-\frac{\rho^{n+1}}{\tau}+\mu s^{n+1} .
$$

We note here that $\phi^{n+1}$ is not concerned variable any more, because it is replaced by variable $\rho^{n+1}$ and $s^{n+1}$ in (1.3) and (1.5), respectively.

The treating on the convection term is a sensitive issue for stability condition, computing cost, and applicability to more complicate fluid problems. There are many numerical treatments for the convection term and we can use any techniques in (1.7). In this paper, we impose the semi-implicit scheme, namely $\left(\mathbf{u}^{n} \cdot \nabla\right) \widehat{\mathbf{u}}^{n+1}$, to make the stability result in [11] satisfy: the gauge-Uzawa Algorithm 1 is unconditionally stable in the sense that, for all $\tau>0$, the following a priori bound holds:

$$
\begin{aligned}
& \left\|\mathbf{u}^{N+1}\right\|_{0}^{2}+\sum_{n=0}^{N}\left\|\mathbf{u}^{n+1}-\mathbf{u}^{n}\right\|_{0}^{2}+\frac{\mu \tau}{2} \sum_{n=0}^{N}\left\|\nabla \widehat{\mathbf{u}}^{n+1}\right\|_{0}^{2} \\
& +2 \sum_{n=0}^{N}\left\|\nabla \rho^{n+1}\right\|_{0}^{2}+\mu \tau\left\|s^{N+1}\right\|_{0}^{2} \\
& \leq\left\|\mathbf{u}^{0}\right\|_{0}^{2}+C \tau \sum_{n=0}^{N}\left\|\mathbf{f}\left(t^{n+1}\right)\right\|_{-1}^{2} .
\end{aligned}
$$

In [11], the convergence results are obtained via the energy estimates as follows: if A1-3 below hold, then we have the error estimates for velocity

$$
\begin{gathered}
\tau \sum_{n=0}^{N}\left\|\nabla\left(\mathbf{u}\left(t^{n+1}\right)-\widehat{\mathbf{u}}^{n+1}\right)\right\|_{0}^{2} \leq C \tau \\
\tau \sum_{n=0}^{N}\left(\left\|\mathbf{u}\left(t^{n+1}\right)-\mathbf{u}^{n+1}\right\|_{0}^{2}+\left\|\mathbf{u}\left(t^{n+1}\right)-\widehat{\mathbf{u}}^{n+1}\right\|_{0}^{2}\right) \leq C \tau^{2},
\end{gathered}
$$

and for pressure and time-derivative of velocity; if A1-4 below hold, then the following error estimates are valid

$$
\tau \sum_{n=0}^{N}\left(\left\|\delta \mathbf{u}\left(t^{n+1}\right)-\delta \mathbf{u}^{n+1}\right\|_{0}^{2}+\left\|p\left(t^{n+1}\right)-p^{n+1}\right\|_{0}^{2}\right) \leq C \tau,
$$

where $\delta$ is the discrete time derivative for a sequence $\left\{W^{n}\right\}_{n=0}^{N}$ to be

$$
\delta W^{n+1}:=\frac{W^{n+1}-W^{n}}{\tau} .
$$


The suboptimal order in (1.11) and (1.12) is due to mismatching between pressure $p$ in NSE and artificial variables $\rho^{n}$ and $s^{n}$ in Algorithm 1. Duality argument using Stokes operators has been applied to cancel out the mismatching terms and this process derives error analysis in $L^{2}$ for time instead of $L^{\infty}$ for time. In this paper, we introduce an intermediate equation (3.7) in which pressure term is replaced to be well matched with Algorithm 1 by using a heat equation (3.1) in $\S 3$. And we estimate velocity errors by comparing with the intermediate equation (3.7) to get optimal accuracy. To control the solution of the heat equation (3.1), we need additional assumptions A5 in $\S 3$ and A6 in $\S 5$.

We now summarize the results of this paper along with its organization. In $\S 2$, we recall some well known lemmas to use in the proof of main theorems, and introduce an intermediate momentum equation (3.7) in $\S 3$. We will prove the errors of velocity in $\S 4$.

Theorem 1.2 (Error estimate for velocity). Suppose A1-4 hold. If A5 in §3 also hold, then we have

$$
\begin{gathered}
\left\|\mathbf{u}\left(t^{n+1}\right)-\mathbf{u}^{n+1}\right\|_{0}^{2}+\tau \sum_{n=0}^{N}\left\|\nabla\left(\mathbf{u}\left(t^{n+1}\right)-\mathbf{u}^{n+1}\right)\right\|_{0}^{2} \leq C \tau^{2}, \\
\left\|\mathbf{u}\left(t^{n+1}\right)-\widehat{\mathbf{u}}^{n+1}\right\|_{0}^{2}+\tau \sum_{n=0}^{N}\left\|\nabla\left(\mathbf{u}\left(t^{n+1}\right)-\widehat{\mathbf{u}}^{n+1}\right)\right\|_{0}^{2} \leq C \tau^{2} .
\end{gathered}
$$

In addition, the following results will be proved in $\S 5$.

Theorem 1.3 (Error estimates for time-derivative of velocity and for pressure). Suppose A1-4 hold. If $\mathrm{A} 6$ in $\S 5$ also hold, then we have

$$
\left\|\delta \mathbf{u}\left(t^{n+1}\right)-\delta \mathbf{u}^{n+1}\right\|_{0}+\left\|\delta \mathbf{u}\left(t^{n+1}\right)-\delta \widehat{\mathbf{u}}^{n+1}\right\|_{0} \leq C \tau .
$$

Finally, the error of pressure will be derived in $\S 6$.

$$
\left\|p\left(t^{n+1}\right)-p^{n+1}\right\|_{0} \leq C \tau \text {. }
$$

\section{Preliminaries}

This section is mainly devoted to reviewing some well-known lemmas. Let $H^{s}(\Omega)$ be the Sobolev space with $s$ derivatives in $L^{2}(\Omega), \mathbf{L}^{2}(\Omega)=\left(L^{2}(\Omega)\right)^{d}$ and $\mathbf{H}^{s}(\Omega)=\left(H^{s}(\Omega)\right)^{d}$, where $d=2,3$. Let $\|\cdot\|_{0}$ denote the $\mathbf{L}^{2}(\Omega)$ norm, and $\langle\cdot, \cdot\rangle$ the corresponding inner product. Let $\|\cdot\|_{s}$ denote the norm of $H^{s}(\Omega)$ for $s \in \mathbb{R}$.

We start with three basic assumptions about $\Omega, \mathbf{u}^{0}, \mathbf{f}$, and $\mathbf{u}$. We consider first the stationary Stokes equations:

$$
\begin{array}{rlrl}
-\triangle \mathbf{w}+\nabla r=\mathbf{g}, & & \text { in } \Omega, \\
\nabla \cdot \mathbf{w}=0, & \text { in } \Omega, \\
\mathbf{w}=\mathbf{0}, & & \text { on } \partial \Omega .
\end{array}
$$


Assumption 1 (Regularity of $(\mathbf{w}, r)$ ). The unique solution $(\mathbf{w}, r) \in \mathbf{H}_{0}^{1}(\Omega) \times$ $L_{0}^{2}(\Omega)$ of the stationary Stokes equations (2.1) satisfies

$$
\|\mathbf{w}\|_{2}+\|r\|_{1} \leq C\|\mathbf{g}\|_{0} .
$$

We remark that A1 is valid provided $\partial \Omega$ is of class $C^{2}[3]$, or if $\Omega$ is a convex two-dimensional polygon [8] or three-dimensional polyhedron [4].

Assumption 2 (Data regularity). The initial velocity $\mathbf{u}^{0}$ and the forcing term f in (1.1) satisfy

$$
\mathbf{u}^{0} \in \mathbf{H}^{2}(\Omega) \cap \mathbf{Z} \quad \text { and } \quad \mathbf{f}, \mathbf{f}_{t} \in L^{\infty}\left(0, T ; \mathbf{L}^{2}(\Omega)\right),
$$

where $\mathbf{Z}:=\left\{\mathbf{z} \in \mathbf{H}_{0}^{1}(\Omega): \nabla \cdot \mathbf{z}=0\right\}$.

Assumption 3 (Regularity of the solution $\mathbf{u}$ ). There exists $M>0$ such that

$$
\sup _{t \in[0, T]}\|\nabla \mathbf{u}(t)\|_{0} \leq M
$$

We note that A3 is always satisfied in $2 \mathrm{~d}$, whereas it is valid in $3 \mathrm{~d}$ provided $\left\|\mathbf{u}^{0}\right\|_{1}$ and $\|\mathbf{f}\|_{L^{\infty}\left(0, T ; \mathbf{L}^{2}(\Omega)\right)}$ are sufficiently small [7].

Lemma 2.1 (Uniform and weighted a priori estimates [7]). Let $\sigma(t)=\min \{t, 1\}$ be a weight function and $0<T \leq \infty$. If A1-3 hold, the solution (u,p) of (1.1) satisfies

$$
\sup _{0<t<T}\left(\|\mathbf{u}\|_{2}+\left\|\mathbf{u}_{t}\right\|_{0}+\|p\|_{1}\right) \leq M, \quad \int_{0}^{T}\left\|\mathbf{u}_{t}\right\|_{1}^{2} d t \leq M,
$$

and

$$
\sup _{0<t<T}\left(\sigma(t)\left\|\mathbf{u}_{t}\right\|_{1}^{2}\right) \leq M, \quad \int_{0}^{T} \sigma(t)\left(\left\|\mathbf{u}_{t}\right\|_{2}^{2}+\left\|\mathbf{u}_{t t}\right\|_{0}^{2}+\left\|p_{t}\right\|_{1}^{2}\right) d t \leq M .
$$

Consequently, $(\mathbf{u}, p) \in L^{\infty}\left(0, T ; \mathbf{H}^{2}(\Omega) \times H^{1}(\Omega)\right)$ provided A1-3 are valid. The following A4 is used to remove the weight $\sigma(t)$ for the error estimates.

Assumption 4 (Nonlocal compatibility). Let $\mathbf{u}^{0}$ and $\mathbf{f}^{0}=\mathbf{f}(0, \cdot)$ be such that

$$
\left\|\nabla \mathbf{u}_{t}(0)\right\|_{0} \leq M
$$

The following lemma is proved in [7].

Lemma 2.2 (Uniform a priori estimates). Suppose A1-3 hold and let $0<T \leq$ $\infty$. Then A4 is valid if and only if

$$
\int_{0}^{T}\left\|\mathbf{u}_{t t}(t)\right\|_{0}^{2} d t+\sup _{0<t<T}\left\|\nabla \mathbf{u}_{t}(t)\right\|_{0}^{2} \leq M
$$

Furthermore, if A4 holds, then $\int_{0}^{T}\left(\left\|p_{t}(t)\right\|_{1}^{2}+\left\|\mathbf{u}_{t}(t)\right\|_{2}^{2}\right) d t \leq M$. 

(1.1)

We now define the trilinear form $\mathcal{N}$ associated with the convection term in

$$
\mathcal{N}(\mathbf{u}, \mathbf{v}, \mathbf{w}):=\int_{\Omega}(\mathbf{u} \cdot \nabla) \mathbf{v} \cdot \mathbf{w} d \mathbf{x},
$$

for which the following properties are well-known [6].

Lemma 2.3 (Properties of $\mathcal{N})$. Let $\mathbf{u}, \mathbf{v}, \mathbf{w} \in \mathbf{H}^{1}(\Omega)$ and $\nabla \cdot \mathbf{u}=0$. If

$$
\mathbf{u} \cdot \boldsymbol{\nu}=0 \quad \text { or } \quad \mathbf{v}=\mathbf{0} \quad \text { on } \partial \Omega,
$$

then

$$
\mathcal{N}(\mathbf{u}, \mathbf{v}, \mathbf{w})=-\mathcal{N}(\mathbf{u}, \mathbf{w}, \mathbf{v}) \quad \text { and } \quad \mathcal{N}(\mathbf{u}, \mathbf{v}, \mathbf{v})=0 .
$$

Sobolev imbedding lemma yields the following results, which will be used later in dealing with the convection term of (1.1).

Lemma 2.4 (Bounds on trilinear form).

$$
\int_{\Omega} \mathbf{u} \cdot \mathbf{v} \cdot \mathbf{w} d \mathbf{x} \leq C\left\{\begin{array}{l}
\|\mathbf{u}\|_{0}\|\mathbf{v}\|_{1}\|\mathbf{w}\|_{1} \\
\|\mathbf{u}\|_{2}\|\mathbf{v}\|_{0}\|\mathbf{w}\|_{0}
\end{array}\right.
$$

The following elementary but crucial relation is derived in $[9,14]$.

Lemma 2.5 (Div-Grad relation). If $\mathbf{v} \in \mathbf{H}_{0}^{1}(\Omega)$, then

$$
\|\nabla \cdot \mathbf{v}\|_{0} \leq\|\nabla \mathbf{v}\|_{0} \text {. }
$$

\section{The intermediate equations}

In this section, we define an intermediate momentum equation and evaluate its error by comparing with (1.1) to use as a reference equation. We start to construct the intermediate momentum equation with defining $\Phi$ as the solution of the heat equation:

$$
\begin{aligned}
\Phi_{t}-\mu \triangle \Phi & =-p, & & \text { in } \Omega, \\
\Phi(0, \mathbf{x}) & =0, & & \text { in } \Omega, \\
\partial_{\boldsymbol{\nu}} \Phi & =0, & & \text { on } \partial \Omega .
\end{aligned}
$$

Let $(\mathbf{v}, q)$ be the solution of Stokes equations

$$
\begin{aligned}
\mathbf{v}_{t}+\nabla q-\triangle \mathbf{v} & =\mathbf{0}, & & \text { in } \Omega, \\
\nabla \cdot \mathbf{v} & =0, & & \text { in } \Omega, \\
\mathbf{v}(0) & =\mathbf{0}, & & \text { in } \Omega, \\
\mathbf{v} & =\varepsilon \nabla \Phi_{t}, & & \text { on } \partial \Omega .
\end{aligned}
$$

From [6], we know that there exists a unique pair $(\mathbf{v}, q)$ solution of $(3.2)$ because of the boundary condition $\partial_{\nu} \Phi_{t}=0$. In addition, we can readily get: 
Lemma 3.1. If $\mathbf{v}$ is the solution of (3.2), then

$$
\|\nabla \mathbf{v}(T)\|_{0}^{2}+\int_{0}^{T}\left\|\mathbf{v}_{t}(t)\right\|_{0}^{2} d t \leq C \varepsilon^{2} \int_{0}^{T}\left(\left\|\Phi_{t t}(t)\right\|_{\frac{1}{2}, \Gamma}^{2}+\left\|\nabla \Phi_{t}(t)\right\|_{\frac{3}{2}, \Gamma}^{2}\right) d t .
$$

In order to use (3.3) in proof of main theorems, we need an additional assumption.

Assumption 5 (Regularity for $\Phi$ ). There exists $M>0$ such that

$$
\int_{0}^{T}\left(\left\|\Phi_{t t}(t)\right\|_{\frac{1}{2}, \Gamma}^{2}+\left\|\nabla \Phi_{t}(t)\right\|_{\frac{3}{2}, \Gamma}^{2}\right) d t \leq M .
$$

We now denote a new variable $\widehat{\mathbf{v}}$ as a solution of the heat equation

$$
\begin{aligned}
\widehat{\mathbf{v}}_{t}-\triangle \widehat{\mathbf{v}} & =-\nabla\left(q+\varepsilon \Phi_{t t}-\varepsilon \Delta \Phi_{t}\right), & & \text { in } \Omega, \\
\widehat{\mathbf{v}}(0) & =\mathbf{0}, & & \text { in } \Omega, \\
\widehat{\mathbf{v}} & =\mathbf{0}, & & \text { on } \partial \Omega,
\end{aligned}
$$

where $q$ is the pressure on (3.2). Then the solutions of (3.2) and (3.4) satisfy

$$
\widehat{\mathbf{v}}:=\mathbf{v}-\varepsilon \nabla \Phi_{t},
$$

and so, in conjunction with A5 and (3.3), we arrive at

$$
\|\nabla \widehat{\mathbf{v}}\|_{0}+\|\nabla \mathbf{v}\|_{0} \leq C \varepsilon
$$

We now define intermediate variables as

$$
\widehat{\mathbf{U}}:=\mathbf{u}+\widehat{\mathbf{v}} \quad \text { and } \quad \mathbf{U}:=\mathbf{u}+\mathbf{v} .
$$

Then divergence free property of $\mathbf{u}$ yields the following lemmas:

Lemma 3.2 (Properties of $\widehat{\mathbf{U}}$ and $\widehat{\mathbf{v}}$ ).

$$
\begin{gathered}
\nabla \cdot \widehat{\mathbf{U}}=\nabla \cdot \widehat{\mathbf{v}}=-\tau \Delta \Phi_{t}, \\
\widehat{\mathbf{U}}=\widehat{\mathbf{v}}=\mathbf{0}, \text { on } \partial \Omega .
\end{gathered}
$$

Lemma 3.3 (Properties of $\mathbf{U}$ and $\mathbf{v}$ ).

$$
\begin{gathered}
\nabla \cdot \mathbf{U}=\nabla \cdot \mathbf{v}=0, \\
\mathbf{U} \cdot \boldsymbol{\nu}=\mathbf{v} \cdot \boldsymbol{\nu}=\partial_{\boldsymbol{\nu}} \Phi_{t}=0, \text { on } \partial \Omega .
\end{gathered}
$$

In order to define the intermediate momentum equation, we fix $\varepsilon=\tau$ from now.

Lemma 3.4 (Intermediate momentum equation). The functions $\widehat{\mathbf{U}}$ and $\mathbf{U}$ satisfy

$$
\begin{gathered}
\frac{\widehat{\mathbf{U}}\left(t^{n+1}\right)-\mathbf{U}\left(t^{n}\right)}{\tau}+\left(\mathbf{u}\left(t^{n+1}\right) \cdot \nabla\right) \mathbf{u}\left(t^{n+1}\right)+\mu \triangle \nabla \Phi\left(t^{n+1}\right) \\
-\mu \triangle \widehat{\mathbf{U}}\left(t^{n+1}\right)=\mathbf{f}\left(t^{n+1}\right)+\mathbf{R}^{n+1}+\mathbf{Q}^{n+1}
\end{gathered}
$$


where

$$
\mathbf{R}^{n+1}:=-\frac{1}{\tau} \int_{t^{n}}^{t^{n+1}}\left(t-t^{n}\right) \mathbf{u}_{t t}(t) d t, \quad \mathbf{Q}^{n+1}:=\delta \mathbf{v}\left(t^{n+1}\right)-\mu \triangle \widehat{\mathbf{v}}\left(t^{n+1}\right) .
$$

In view of (3.5) and (3.6), we arrive at:

Lemma 3.5 (Error estimates of $\mathbf{U}$ and $\widehat{\mathbf{U}})$. If A1-5 hold, then we have

$$
\|\mathbf{u}-\mathbf{U}\|_{1}+\|\mathbf{u}-\widehat{\mathbf{U}}\|_{1} \leq C \tau \text {. }
$$

\section{Error analysis for velocity}

In this section, we carry out error estimate for velocity. Since we have Lemma 3.5, it is enough to estimate $\left\|\mathbf{U}^{n+1}-\mathbf{u}^{n+1}\right\|_{0} \leq C \tau$. In this proof, we use that $\left(\widehat{\mathbf{u}}^{n+1}, \mathbf{u}^{n+1}, p^{n+1}, s^{n+1}, \rho^{n+1}\right)$ as the solution of the Algorithm 1 and denote the corresponding errors by

$$
\begin{array}{ll}
\widehat{\mathbf{E}}^{n+1}:=\widehat{\mathbf{U}}\left(t^{n+1}\right)-\widehat{\mathbf{u}}^{n+1}, & \varepsilon^{n+1}:=\triangle \Phi\left(t^{n+1}\right)-s^{n+1}, \\
\mathbf{E}^{n+1}:=\mathbf{U}\left(t^{n+1}\right)-\mathbf{u}^{n+1}, & \gamma^{n+1}:=\rho^{n+1}-\tau \Phi_{t}\left(t^{n+1}\right) .
\end{array}
$$

Before embarking on this discussion, we mention several useful properties of the error functions. In view of Lemmas 3.2 and 3.3, we have

$$
\begin{aligned}
& \nabla \cdot \mathbf{E}^{n+1}=0, \quad \widehat{\mathbf{E}}^{n+1}=\mathbf{E}^{n+1}+\nabla \gamma^{n+1}, \\
& \mathbf{E}^{n+1} \cdot \boldsymbol{\nu}=\mathbf{0} \quad \text { and } \quad \widehat{\mathbf{E}}^{n+1}=\mathbf{0} \quad \text { on } \partial \Omega .
\end{aligned}
$$

In conjunction with (1.8), Lemma 3.2 derives

$$
\begin{aligned}
\nabla \cdot \widehat{\mathbf{E}}^{n+1} & =-\tau \triangle \Phi_{t}\left(t^{n+1}\right)+s^{n+1}-s^{n} \\
& =-\int_{t^{n}}^{t^{n+1}}\left(t-t^{n}\right) \triangle \Phi_{t t}(t) d t-\varepsilon^{n+1}+\varepsilon^{n} .
\end{aligned}
$$

If we apply $\mathbf{U}(0)=\widehat{\mathbf{U}}(0)=\mathbf{u}(0)$, then we readily get

$$
\mathbf{E}^{0}=\widehat{\mathbf{E}}^{0}=\mathbf{0} \text {. }
$$

As well as, (4.1) derives

$$
\begin{aligned}
\left\|\mathbf{E}^{n+1}\right\|_{0}^{2} & =\left\langle\widehat{\mathbf{E}}^{n+1}, \mathbf{E}^{n+1}\right\rangle \\
\left\|\widehat{\mathbf{E}}^{n+1}\right\|_{0}^{2} & =\left\|\mathbf{E}^{n+1}\right\|_{0}^{2}+\left\|\nabla \gamma^{n+1}\right\|_{0}^{2} .
\end{aligned}
$$

We use (4.1) again and apply Lemma 2.5 to obtain

and

$$
\left\|\Delta \gamma^{n+1}\right\|_{0}^{2}=\left\|\nabla \cdot \widehat{\mathbf{E}}^{n+1}\right\|_{0}^{2} \leq\left\|\nabla \widehat{\mathbf{E}}^{n+1}\right\|_{0}^{2}
$$

$$
\left\|\nabla \mathbf{E}^{n+1}\right\|_{0}^{2} \leq C\left\|\nabla \widehat{\mathbf{E}}^{n+1}\right\|_{0}^{2}+C\left\|\triangle \gamma^{n+1}\right\|_{0}^{2} \leq 2 C\left\|\nabla \widehat{\mathbf{E}}^{n+1}\right\|_{0}^{2}
$$


In light of the definition of $\varepsilon^{n+1}$ in (4.1), applying (4.2) and Lemma 2.5 yield

$$
\begin{aligned}
\left\|\varepsilon^{n+1}-\varepsilon^{n}\right\|_{0}^{2} & =\left\|\triangle\left(\Phi\left(t^{n+1}\right)-\Phi\left(t^{n}\right)\right)-\left(s^{n+1}-s^{n}\right)\right\|_{0}^{2} \\
& \leq C \tau^{3} \int_{t^{n}}^{t^{n+1}}\left\|\triangle \Phi_{t t}(t)\right\|_{0}^{2} d t+\frac{21}{20}\left\|\nabla \widehat{\mathbf{E}}^{n+1}\right\|_{0}^{2} .
\end{aligned}
$$

We now start to estimate error of velocity.

Lemma 4.1 (Estimate $\left.\left\|\mathbf{E}^{n+1}\right\|_{0}\right)$. Let Assumptions A1-5 hold. Then we have

$$
\begin{aligned}
& \left\|\widehat{\mathbf{E}}^{n+1}\right\|_{0}^{2}+\left\|\mathbf{E}^{n+1}\right\|_{0}^{2}+\sum_{n=0}^{N}\left\|\mathbf{E}^{n+1}-\mathbf{E}^{n}\right\|_{0}^{2}+2 \sum_{n=0}^{N}\left\|\nabla \gamma^{n+1}\right\|_{0}^{2} \\
& +\frac{\mu}{2} \tau \sum_{n=0}^{N}\left(\left\|\nabla \widehat{\mathbf{E}}^{n+1}\right\|_{0}^{2}+\left\|\nabla \mathbf{E}^{n+1}\right\|_{0}^{2}\right)+\tau \mu\left\|\varepsilon^{N+1}\right\|_{0}^{2} \leq C \tau^{2} .
\end{aligned}
$$

Proof. We subtract (1.7) from (3.7) to get

$$
\begin{aligned}
\frac{\widehat{\mathbf{E}}^{n+1}-\mathbf{E}^{n}}{\tau}-\mu \triangle \widehat{\mathbf{E}}^{n+1}= & \mathbf{R}^{n+1}+\mathbf{Q}^{n+1}-\mu \nabla\left(\triangle \Phi\left(t^{n+1}\right)-s^{n}\right) \\
& -\left(\mathbf{u}\left(t^{n+1}\right) \cdot \nabla\right) \mathbf{u}\left(t^{n+1}\right)+\left(\mathbf{u}^{n} \cdot \nabla\right) \widehat{\mathbf{u}}^{n+1}
\end{aligned}
$$

Multiplying (4.7) by $2 \tau \widehat{\mathbf{E}}^{n+1} \in \mathbf{H}_{0}^{1}(\Omega)$ and invoking (4.3) yields

$$
\left\|\mathbf{E}^{n+1}\right\|_{0}^{2}-\left\|\mathbf{E}^{n}\right\|_{0}^{2}+\left\|\mathbf{E}^{n+1}-\mathbf{E}^{n}\right\|_{0}^{2}+2 \mu \tau\left\|\nabla \widehat{\mathbf{E}}^{n+1}\right\|_{0}^{2}+2\left\|\nabla \gamma^{n+1}\right\|_{0}^{2}=\sum_{i=1}^{3} A_{i}
$$

where

$$
\begin{aligned}
& A_{1}:=2 \tau\left\langle\mathbf{R}^{n+1}+\mathbf{Q}^{n+1}, \widehat{\mathbf{E}}^{n+1}\right\rangle, \\
& A_{2}:=2 \mu \tau\left\langle\triangle \Phi\left(t^{n+1}\right)-s^{n}, \nabla \cdot \widehat{\mathbf{E}}^{n+1}\right\rangle, \\
& A_{3}:=2 \tau\left(\mathcal{N}\left(\mathbf{u}^{n}, \widehat{\mathbf{u}}^{n+1}, \widehat{\mathbf{E}}^{n+1}\right)-\mathcal{N}\left(\mathbf{u}\left(t^{n+1}\right), \mathbf{u}\left(t^{n+1}\right), \widehat{\mathbf{E}}^{n+1}\right)\right) .
\end{aligned}
$$

We now estimate terms $A_{1}$ to $A_{3}$ separately. We use the Hölder inequality to get

$A_{1} \leq \frac{\mu}{20} \tau\left\|\nabla \widehat{\mathbf{E}}^{n+1}\right\|_{0}^{2}+C \mu \tau\left\|\nabla \widehat{\mathbf{v}}\left(t^{n+1}\right)\right\|_{0}^{2}+\frac{C}{\mu} \int_{t^{n}}^{t^{n+1}}\left(\tau^{2}\left\|\mathbf{u}_{t t}(t)\right\|_{-1}^{2}+\left\|\mathbf{v}_{t}(t)\right\|_{0}^{2}\right) d t$.

On employing (4.2), $A_{2}$ becomes

$$
\begin{aligned}
A_{2}= & 2 \mu \tau\left\langle\triangle\left(\Phi\left(t^{n+1}\right)-\Phi\left(t^{n}\right)\right)+\varepsilon^{n},-\int_{t^{n}}^{t^{n+1}}\left(t-t^{n}\right) \triangle \Phi_{t t}(t) d t-\varepsilon^{n+1}+\varepsilon^{n}\right\rangle \\
\leq & -2 \tau \mu\left\langle\varepsilon^{n}, \varepsilon^{n+1}-\varepsilon^{n}\right\rangle+\frac{\mu \tau}{20}\left\|\varepsilon^{n+1}-\varepsilon^{n}\right\|_{0}^{2}+C \tau^{2} \mu\left\|\varepsilon^{n}\right\|_{0}^{2} \\
& +C \tau^{2} \mu \int_{t^{n}}^{t^{n+1}}\left(\left\|\triangle \Phi_{t}(t)\right\|_{0}^{2}+\left\|\triangle \Phi_{t t}(t)\right\|_{0}^{2}\right) d t .
\end{aligned}
$$


If we apply $-2\left\langle\varepsilon^{n}, \varepsilon^{n+1}-\varepsilon^{n}\right\rangle=-\left(\left\|\varepsilon^{n+1}\right\|_{0}^{2}-\left\|\varepsilon^{n}\right\|_{0}^{2}-\left\|\varepsilon^{n+1}-\varepsilon^{n}\right\|_{0}^{2}\right)$ in conjunction with (4.5), then we obtain

$$
\begin{aligned}
A_{2} \leq & -\tau \mu\left(\left\|\varepsilon^{n+1}\right\|_{0}^{2}-\left\|\varepsilon^{n}\right\|_{0}^{2}\right)+C \tau^{2} \mu\left\|\varepsilon^{n}\right\|_{0}^{2}+\frac{22 \mu \tau}{20}\left\|\nabla \widehat{\mathbf{E}}^{n+1}\right\|_{0}^{2} \\
& +C \mu \tau^{2} \int_{t^{n}}^{t^{n+1}}\left(\left\|\triangle \Phi_{t}(t)\right\|_{0}^{2}+\left\|\triangle \Phi_{t t}(t)\right\|_{0}^{2}\right) d t .
\end{aligned}
$$

At the same time, the convection term $A_{3}$ can be rewritten as $A_{3}=A_{3,1}+A_{3,2}$ with

$$
\begin{aligned}
& A_{3,1}:=-2 \tau \mathcal{N}\left(\mathbf{u}\left(t^{n+1}\right)-\mathbf{u}\left(t^{n}\right)-\mathbf{v}\left(t^{n}\right)+\mathbf{E}^{n}, \mathbf{u}\left(t^{n+1}\right), \widehat{\mathbf{E}}^{n+1}\right), \\
& A_{3,2}:=-2 \tau \mathcal{N}\left(\mathbf{u}^{n}, \widehat{\mathbf{E}}^{n+1}-\widehat{\mathbf{v}}\left(t^{n+1}\right), \widehat{\mathbf{E}}^{n+1}\right) .
\end{aligned}
$$

Since $\left\|\mathbf{u}\left(t^{n+1}\right)\right\|_{2} \leq M$, Lemma 2.4 gives

$$
\begin{aligned}
A_{3,1} & \leq C \tau\left(\left\|\mathbf{u}\left(t^{n+1}\right)-\mathbf{u}\left(t^{n}\right)\right\|_{0}+\left\|\mathbf{v}\left(t^{n}\right)\right\|_{0}+\left\|\mathbf{E}^{n}\right\|_{0}\right)\left\|\mathbf{u}\left(t^{n+1}\right)\right\|_{2}\left\|\nabla \widehat{\mathbf{E}}^{n+1}\right\|_{0}^{2} \\
& \leq \frac{C}{\mu} \tau^{2} \int_{t^{n}}^{t^{n+1}}\left\|\mathbf{u}_{t}(t)\right\|_{0}^{2} d t+\frac{C}{\mu} \tau\left(\left\|\mathbf{v}\left(t^{n}\right)\right\|_{0}^{2}+\left\|\mathbf{E}^{n}\right\|_{0}^{2}\right)+\frac{\mu}{20} \tau\left\|\nabla \widehat{\mathbf{E}}^{n+1}\right\|_{0}^{2} .
\end{aligned}
$$

To estimate $A_{3,2}$, we first note $\mathcal{N}\left(\mathbf{u}^{n}, \widehat{\mathbf{E}}^{n+1}, \widehat{\mathbf{E}}^{n+1}\right)=0$ from Lemma 2.3. We next invoke $\left\|\nabla \widehat{\mathbf{v}}\left(t^{n+1}\right)\right\|_{0} \leq C \tau$, which comes from (3.5), to get

$$
\begin{aligned}
A_{3,2} & =2 \tau \mathcal{N}\left(\mathbf{u}\left(t^{n}\right)-\mathbf{v}\left(t^{n}\right)-\mathbf{E}^{n}, \widehat{\mathbf{v}}\left(t^{n+1}\right), \widehat{\mathbf{E}}^{n+1}\right) \\
& \leq C \tau\left(\left\|\mathbf{u}\left(t^{n}\right)\right\|_{2}\left\|\widehat{\mathbf{v}}\left(t^{n+1}\right)\right\|_{0}+\left\|\nabla\left(\mathbf{v}\left(t^{n}\right)+\mathbf{E}^{n}\right)\right\|_{0}\left\|\nabla \widehat{\mathbf{v}}\left(t^{n+1}\right)\right\|_{0}\right)\left\|\nabla \widehat{\mathbf{E}}^{n+1}\right\|_{0} \\
& \leq \frac{C}{\mu} \tau\left\|\widehat{\mathbf{v}}\left(t^{n+1}\right)\right\|_{0}^{2}+\frac{C}{\mu} \tau^{3}\left(\left\|\nabla \mathbf{v}\left(t^{n}\right)\right\|_{0}^{2}+\left\|\nabla \mathbf{E}^{n}\right\|_{0}^{2}\right)+\frac{\mu}{20} \tau\left\|\nabla \widehat{\mathbf{E}}^{n+1}\right\|_{0}^{2} \cdot
\end{aligned}
$$

Inserting the above estimates $A_{1}-A_{3}$ into (4.8) and summing over $n$ from 0 to $N$ lead to

$$
\begin{aligned}
& \left\|\mathbf{E}^{N+1}\right\|_{0}^{2}+\sum_{n=0}^{N}\left\|\mathbf{E}^{n+1}-\mathbf{E}^{n}\right\|_{0}^{2}+\frac{3 \mu}{4} \tau \sum_{n=0}^{N}\left\|\nabla \widehat{\mathbf{E}}^{n+1}\right\|_{0}^{2} \\
& +\mu \tau\left\|\varepsilon^{N+1}\right\|_{0}^{2}+2 \sum_{n=0}^{N}\left\|\nabla \gamma^{n+1}\right\|_{0}^{2} \\
& \leq C \mu \tau \sum_{n=0}^{N}\left\|\nabla \widehat{\mathbf{v}}\left(t^{n+1}\right)\right\|_{0}^{2}+C \mu \tau^{2} \sum_{n=0}^{N}\left\|\varepsilon^{n}\right\|_{0}^{2} \\
& +\frac{C}{\mu} \tau \sum_{n=0}^{N}\left(\left\|\mathbf{E}^{n}\right\|_{0}^{2}+\left\|\mathbf{v}\left(t^{n}\right)\right\|_{0}^{2}+\left\|\widehat{\mathbf{v}}\left(t^{n+1}\right)\right\|_{0}^{2}\right)+\frac{C}{\mu} \tau^{3}\left(\left\|\nabla \mathbf{E}^{n}\right\|_{0}^{2}+\left\|\nabla \mathbf{v}\left(t^{n}\right)\right\|_{0}^{2}\right) \\
& +C \tau^{2} \int_{t^{0}}^{t^{N+1}}\left(\left\|\mathbf{u}_{t}(t)\right\|_{0}^{2}+\left\|\mathbf{u}_{t t}(t)\right\|_{-1}^{2}+\left\|\triangle \Phi_{t t}(t)\right\|_{0}^{2}+\left\|\mathbf{v}_{t}(t)\right\|_{0}^{2}\right) d t .
\end{aligned}
$$


On employing (4.4), $\frac{C}{\mu} \tau^{3} \sum_{n=0}^{N}\left\|\nabla \mathbf{E}^{n}\right\|_{0}^{2}$ can be canceled with

$$
\frac{\mu}{4} \sum_{n=0}^{N}\left\|\nabla \widehat{\mathbf{E}}^{n+1}\right\|_{0}^{2}
$$

if $\tau$ is small enough. Finally, we apply Lemma 3.1 and the discrete Gronwall lemma to obtain (4.6) and to finish this proof.

\section{Error analysis for time-derivative of velocity}

In this section, we carry out the error analysis for the time-derivative of velocity which is stated in (1.13) of Theorem 1.3. To do this, we need to use the following lemma.

Lemma 5.1. If $\mathbf{v}$ is the solution of (3.2), then we have

$\left\|\nabla \mathbf{v}_{t}\right\|_{0}^{2}+\int_{0}^{T}\left\|\mathbf{v}_{t t}\right\|_{0}^{2} d t \leq C \varepsilon^{2}\left(\left\|\Phi_{t t}(0)\right\|_{2}^{2}+\int_{0}^{T}\left(\left\|\Phi_{t t t}\right\|_{\frac{1}{2}, \Gamma}^{2}+\left\|\nabla \Phi_{t t}\right\|_{\frac{3}{2}, \Gamma}^{2}\right) d t\right)$.

In order to use (5.1) in the proof of main theorems and treat high order time derivative velocity terms, we need rather strong regularity assumption.

Assumption 6 (Additional regularity for $\Phi$ and $\mathbf{u}$ ). There exists $M>0$ such that

$$
\begin{gathered}
\max _{0 \leq t \leq \tau}\left(\left\|\nabla \triangle \Phi_{t}(t)\right\|_{0}^{2}+\left\|\mathbf{u}_{t t}(t)\right\|_{0}^{2}+\left\|\Phi_{t t}(t)\right\|_{2}^{2}\right) \leq M, \\
\int_{0}^{T}\left(\left\|\Phi_{t t t}\right\|_{\frac{1}{2}, \Gamma}^{2}+\left\|\nabla \Phi_{t t}\right\|_{\frac{3}{2}, \Gamma}^{2}+\left\|\mathbf{u}_{t t t}\right\|_{-1}^{2}\right) d t \leq M .
\end{gathered}
$$

We start to evaluate errors at $t=t^{1}$. It will be utilize in Lemma 5.3.

Lemma 5.2 (Initial errors). Suppose A1-6 hold. Then we have

$$
\left\|\widehat{\mathbf{E}}^{1}\right\|_{0}^{2}+\left\|\mathbf{E}^{1}\right\|_{0}^{2}+\mu \tau\left\|\nabla \widehat{\mathbf{E}}^{1}\right\|_{0}^{2}+\left\|\nabla \gamma^{1}\right\|_{0}^{2}+\left\|\varepsilon^{1}\right\|_{0}^{2} \leq C \tau^{4}
$$

Proof. Since $\mathbf{E}^{0}=\mathbf{0}$, (4.8) for the case $n=0$ becomes

$$
\left\|\mathbf{E}^{1}\right\|_{0}^{2}+\mu \tau\left\|\nabla \widehat{\mathbf{E}}^{1}\right\|_{0}^{2}+\left\|\nabla \gamma^{1}\right\|_{0}^{2}=\sum_{i=1}^{3} A_{i}
$$

where

$$
\begin{aligned}
& A_{1}:=\tau\left\langle\mathbf{R}^{1}+\mathbf{Q}^{1}, \widehat{\mathbf{E}}^{1}\right\rangle \\
& A_{2}:=\mu \tau\left\langle\nabla \triangle \Phi\left(t^{1}\right), \nabla \cdot \widehat{\mathbf{E}}^{1}\right\rangle \\
& A_{3}:=\tau\left(\mathcal{N}\left(\mathbf{u}^{0}, \widehat{\mathbf{u}}^{1}, \widehat{\mathbf{E}}^{1}\right)-\mathcal{N}\left(\mathbf{u}\left(t^{1}\right), \mathbf{u}\left(t^{1}\right), \widehat{\mathbf{E}}^{1}\right)\right) .
\end{aligned}
$$

We now estimate terms $A_{1}$ to $A_{3}$ separately. We first remark

$$
\left\|\widehat{\mathbf{v}}\left(t^{1}\right)\right\|_{1}^{2}=\left\|\widehat{\mathbf{v}}\left(t^{1}\right)-\widehat{\mathbf{v}}(0)\right\|_{1}^{2} \leq C \tau \int_{0}^{\tau}\left\|\widehat{\mathbf{v}}_{t}(t)\right\|_{0}^{2} d t
$$


to estimate $A_{1}$ as

$$
\begin{aligned}
A_{1} \leq & \frac{\mu}{2} \tau\left\|\nabla \widehat{\mathbf{E}}^{1}\right\|_{0}^{2}+\frac{1}{6}\left\|\widehat{\mathbf{E}}^{1}\right\|_{0}^{2}+C \tau^{3} \int_{0}^{\tau}\left\|\mathbf{u}_{t t}(t)\right\|_{0}^{2} d t \\
& +C \tau \int_{0}^{\tau}\left\|\mathbf{v}_{t}(t)\right\|_{0}^{2} d t+C \mu \tau \int_{0}^{\tau}\left\|\nabla \widehat{\mathbf{v}}_{t}(t)\right\|_{0}^{2} d t
\end{aligned}
$$

If we use $\Phi(0)=0$, then we can readily get

$$
A_{2}=2 \mu \tau\left\langle\triangle\left(\Phi\left(t^{1}\right)-\Phi(0)\right), \nabla \cdot \widehat{\mathbf{E}}^{1}\right\rangle \leq C \tau^{3} \int_{0}^{\tau}\left\|\nabla \triangle \Phi_{t}(t)\right\|_{0}^{2} d t+\frac{1}{6}\left\|\widehat{\mathbf{E}}^{1}\right\|_{0}^{2}
$$

Since $\mathbf{u}\left(t^{0}\right)=\mathbf{u}^{0}$, Lemmas 2.3 and 2.4 yield

$$
\begin{aligned}
A_{3} & =-2 \tau \mathcal{N}\left(\mathbf{u}\left(t^{1}\right)-\mathbf{u}\left(t^{0}\right), \mathbf{u}\left(t^{1}\right), \widehat{\mathbf{E}}^{1}\right)-2 \tau \mathcal{N}\left(\mathbf{u}\left(t^{0}\right), \widehat{\mathbf{E}}^{1}-\widehat{\mathbf{v}}\left(t^{1}\right), \widehat{\mathbf{E}}^{1}\right) \\
& \leq C \tau\left(\left\|\mathbf{u}\left(t^{1}\right)-\mathbf{u}\left(t^{0}\right)\right\|_{1}\left\|\mathbf{u}\left(t^{1}\right)\right\|_{2}+\left\|\mathbf{u}\left(t^{0}\right)\right\|_{2}\left\|\widehat{\mathbf{v}}\left(t^{1}\right)\right\|_{1}\right)\left\|\widehat{\mathbf{E}}^{1}\right\|_{0} \\
& \leq C \tau^{3} \int_{0}^{\tau}\left\|\mathbf{u}_{t}(t)\right\|_{1}^{2} d t+C \tau^{2}\left\|\widehat{\mathbf{v}}\left(t^{1}\right)\right\|_{1}^{2}+\frac{1}{6}\left\|\widehat{\mathbf{E}}^{1}\right\|_{0}^{2} .
\end{aligned}
$$

Inserting $A_{1}-A_{3}$ into (5.3) and applying (4.3), we arrive at

$$
\left\|\widehat{\mathbf{E}}^{1}\right\|_{0}^{2}+\left\|\mathbf{E}^{1}\right\|_{0}^{2}+\mu \tau\left\|\nabla \widehat{\mathbf{E}}^{1}\right\|_{0}^{2}+\left\|\nabla \gamma^{1}\right\|_{0}^{2} \leq C \tau^{4}
$$

If we know (4.5) and (5.4), then we can readily get $\left\|\varepsilon^{1}\right\|_{0}^{2} \leq C \tau^{2}$ and arrive at $(5.2)$.

We now prove error for time-derivative of velocity.

Lemma 5.3 (Estimate $\left.\left\|\delta \mathbf{E}^{n+1}\right\|_{0}\right)$. Suppose A1-6 hold. Then we have

$$
\begin{gathered}
\left\|\delta \widehat{\mathbf{E}}^{N+1}\right\|_{0}^{2}+\left\|\delta \mathbf{E}^{N+1}\right\|_{0}^{2}+\sum_{n=1}^{N}\left\|\delta \mathbf{E}^{n+1}-\delta \mathbf{E}^{n}\right\|_{0}^{2}+2 \sum_{n=1}^{N}\left\|\nabla \delta \gamma^{n+1}\right\|_{0}^{2} \\
+\frac{\mu}{2} \tau \sum_{n=1}^{N}\left(\left\|\nabla \delta \widehat{\mathbf{E}}^{n+1}\right\|_{0}^{2}+\left\|\nabla \delta \mathbf{E}^{n+1}\right\|_{0}^{2}\right)+\mu \tau\left\|\delta \varepsilon^{n+1}\right\|_{0}^{2} \leq C \tau^{2}
\end{gathered}
$$

Proof. Subtracting two consecutive expressions (4.7), multiplying by $2 \delta \widehat{\mathbf{E}}^{n+1} \in$ $\mathbf{H}_{0}^{1}(\Omega)$, and arguments leading to (4.3), yield

$$
\left\|\delta \mathbf{E}^{n+1}\right\|_{0}^{2}-\left\|\delta \mathbf{E}^{n}\right\|_{0}^{2}+\left\|\delta \mathbf{E}^{n+1}-\delta \mathbf{E}^{n}\right\|_{0}^{2}+2\left\|\nabla \delta \gamma^{n+1}\right\|_{0}^{2}+2 \mu \tau\left\|\nabla \delta \widehat{\mathbf{E}}^{n+1}\right\|_{0}^{2}=\sum_{i=1}^{3} A_{i}
$$


where

$$
\begin{aligned}
A_{1}:= & 2 \tau\left\langle\delta \mathbf{R}^{n+1}+\delta \mathbf{Q}^{n+1}, \delta \widehat{\mathbf{E}}^{n+1}\right\rangle \\
A_{2}:= & 2 \mu \tau\left\langle\triangle \delta \Phi\left(t^{n+1}\right)-\delta s^{n}, \nabla \cdot \delta \widehat{\mathbf{E}}^{n+1}\right\rangle \\
A_{3}:= & -2 \mathcal{N}\left(\mathbf{u}\left(t^{n+1}\right), \mathbf{u}\left(t^{n+1}\right), \delta \widehat{\mathbf{E}}^{n+1}\right)+2 \mathcal{N}\left(\mathbf{u}\left(t^{n}\right), \mathbf{u}\left(t^{n}\right), \delta \widehat{\mathbf{E}}^{n+1}\right), \\
& +2 \mathcal{N}\left(\mathbf{u}^{n}, \widehat{\mathbf{u}}^{n+1}, \delta \widehat{\mathbf{E}}^{n+1}\right)-2 \mathcal{N}\left(\mathbf{u}^{n-1}, \widehat{\mathbf{u}}^{n}, \delta \widehat{\mathbf{E}}^{n+1}\right) .
\end{aligned}
$$

We will estimate $A_{i}$ separately. We can readily get

$A_{1} \leq \frac{\mu}{20} \tau\left\|\nabla \delta \widehat{\mathbf{E}}^{n+1}\right\|_{0}^{2}+\frac{C}{\mu} \int_{t^{n-1}}^{t^{n+1}}\left(\tau^{2}\left\|\mathbf{u}_{t t t}\right\|_{-1}^{2}+\left\|\mathbf{v}_{t t}\right\|_{0}^{2}\right) d t+\frac{C}{\mu} \int_{t^{n}}^{t^{n+1}}\left\|\nabla \mathbf{v}_{t}\right\|_{0}^{2} d t$.

On employing (4.2) and (4.5), $A_{2}$ becomes

$$
\begin{aligned}
A_{2}= & -2 \mu \tau\left\langle\triangle\left(\delta \Phi\left(t^{n+1}\right)-\delta \Phi\left(t^{n}\right)\right)+\delta \varepsilon^{n},-\tau \triangle \delta \Phi_{t}\left(t^{n+1}\right)+\delta\left(s^{n+1}-s^{n}\right)\right\rangle \\
\leq & -\mu \tau\left(\left\|\delta \varepsilon^{n+1}\right\|_{0}^{2}-\left\|\delta \varepsilon^{n}\right\|_{0}^{2}\right)+\frac{23 \mu}{20} \tau\left\|\nabla \delta \widehat{\mathbf{E}}^{n+1}\right\|_{0}^{2}+C \mu \tau^{2}\left\|\delta \varepsilon^{n}\right\|_{0}^{2} \\
& +C \mu \tau^{2} \int_{t^{n-1}}^{t^{n+1}}\left(\left\|\triangle \Phi_{t t}(t)\right\|+\left\|\triangle \Phi_{t t t}(t)\right\|\right) d t .
\end{aligned}
$$

On the other hand, an elementary manipulation rewrite $A_{3}$ as $A_{3}=\sum_{i=1}^{6} A_{3, i}$ with

$$
\begin{aligned}
& A_{3,1}:=-2 \mathcal{N}\left(\mathbf{u}\left(t^{n+1}\right)-2 \mathbf{u}\left(t^{n}\right)+\mathbf{u}\left(t^{n-1}\right), \mathbf{u}\left(t^{n+1}\right), \delta \widehat{\mathbf{E}}^{n+1}\right), \\
& A_{3,2}:=-2 \mathcal{N}\left(\mathbf{u}\left(t^{n}\right)-\mathbf{u}\left(t^{n-1}\right), \widehat{\mathbf{E}}^{n+1}-\widehat{\mathbf{v}}\left(t^{n+1}\right), \delta \widehat{\mathbf{E}}^{n+1}\right), \\
& A_{3,3}:=-2 \mathcal{N}\left(\left(\mathbf{E}^{n}-\mathbf{E}^{n-1}\right)-\left(\mathbf{v}\left(t^{n}\right)-\mathbf{v}\left(t^{n-1}\right)\right), \widehat{\mathbf{u}}^{n+1}, \delta \widehat{\mathbf{E}}^{n+1}\right), \\
& A_{3,4}:=-2 \mathcal{N}\left(\mathbf{E}^{n-1}-\mathbf{v}\left(t^{n-1}\right), \mathbf{u}\left(t^{n+1}\right)-\mathbf{u}\left(t^{n}\right), \delta \widehat{\mathbf{E}}^{n+1}\right), \\
& A_{3,5}:=-2 \mathcal{N}\left(\mathbf{u}^{n-1},\left(\widehat{\mathbf{E}}^{n+1}-\widehat{\mathbf{E}}^{n}\right)-\left(\widehat{\mathbf{v}}\left(t^{n+1}\right)-\widehat{\mathbf{v}}\left(t^{n}\right)\right), \delta \widehat{\mathbf{E}}^{n+1}\right), \\
& A_{3,6}=-2 \mathcal{N}\left(\mathbf{u}\left(t^{n}\right)-\mathbf{u}\left(t^{n-1}\right), \mathbf{u}\left(t^{n+1}\right)-\mathbf{u}\left(t^{n}\right), \delta \widehat{\mathbf{E}}^{n+1}\right) .
\end{aligned}
$$

In estimate $A_{3}$, we will use frequently Lemma 2.4 without notice. Applying $\left\|\mathbf{u}\left(t^{n+1}\right)\right\|_{2} \leq M$, we have

$$
\begin{aligned}
A_{3,1} & \leq C\left\|\mathbf{u}\left(t^{n+1}\right)-2 \mathbf{u}\left(t^{n}\right)+\mathbf{u}\left(t^{n-1}\right)\right\|_{0}\left\|\mathbf{u}\left(t^{n+1}\right)\right\|_{2}\left\|\nabla \delta \widehat{\mathbf{E}}^{n+1}\right\|_{0} \\
& \leq \frac{C}{\mu} \tau^{2} \int_{t^{n-1}}^{t^{n+1}}\left\|\mathbf{u}_{t t}(t)\right\|_{0}^{2} d t+\frac{\mu}{20} \tau\left\|\nabla \delta \widehat{\mathbf{E}}^{n+1}\right\|_{0}^{2} .
\end{aligned}
$$

Since we have $\left\|\widehat{\mathbf{E}}^{n+1}-\widehat{\mathbf{v}}\left(t^{n+1}\right)\right\|_{0} \leq C \tau$ which is the result of Lemma $4.1, A_{3,2}$ becomes

$$
A_{3,2} \leq \frac{C}{\mu} \tau^{2} \int_{t^{n}}^{t^{n+1}}\left\|\mathbf{u}_{t}(t)\right\|_{2}^{2} d t+\frac{\mu}{20} \tau\left\|\nabla \delta \widehat{\mathbf{E}}^{n+1}\right\|_{0}^{2}
$$


Rewriting $\widehat{\mathbf{u}}^{n+1}=\mathbf{u}\left(t^{n+1}\right)+\widehat{\mathbf{v}}\left(t^{n+1}\right)-\widehat{\mathbf{E}}^{n+1}$, applying $\Lambda:=\left\|\widehat{\mathbf{v}}\left(t^{n+1}\right)-\widehat{\mathbf{E}}^{n+1}\right\|_{1}$ $\leq C \tau^{\frac{1}{2}}$ which is obtained from the result of Lemma 4.1 lead us

$$
\begin{aligned}
A_{3,3} \leq & C \tau\left(\left\|\delta \mathbf{E}^{n}-\delta \mathbf{v}\left(t^{n}\right)\right\|_{1} \Lambda+\left\|\delta \mathbf{E}^{n}-\delta \mathbf{v}\left(t^{n}\right)\right\|_{0}\left\|\mathbf{u}\left(t^{n+1}\right)\right\|_{2}\right)\left\|\nabla \delta \widehat{\mathbf{E}}^{n+1}\right\|_{0} \\
\leq & \frac{C}{\mu} \tau^{2}\left(\left\|\nabla \delta \mathbf{E}^{n}\right\|_{0}^{2}+\left\|\nabla \delta \mathbf{v}\left(t^{n}\right)\right\|_{0}^{2}\right)+\frac{C}{\mu} \tau\left(\left\|\delta \mathbf{E}^{n}\right\|_{0}^{2}+\left\|\delta \mathbf{v}\left(t^{n}\right)\right\|_{0}^{2}\right) \\
& +\frac{\mu}{20} \tau\left\|\nabla \delta \widehat{\mathbf{E}}^{n+1}\right\|_{0}^{2} .
\end{aligned}
$$

Likewise, we use $\left\|\mathbf{E}^{n-1}-\mathbf{v}\left(t^{n-1}\right)\right\|_{0} \leq C \tau$ again to get

$$
A_{3,4} \leq \frac{C}{\mu} \tau^{2} \int_{t^{n}}^{t^{n+1}}\left\|\mathbf{u}_{t}(t)\right\|_{0}^{2} d t+\frac{\mu}{20} \tau\left\|\nabla \delta \widehat{\mathbf{E}}^{n+1}\right\|_{0}^{2} .
$$

We note here $\mathcal{N}\left(\mathbf{u}^{n-1}, \delta \widehat{\mathbf{E}}^{n+1}, \delta \widehat{\mathbf{E}}^{n+1}\right)=0$ and $\mathbf{u}^{n-1}=\mathbf{u}\left(t^{n-1}\right)-\widehat{\mathbf{E}}^{n-1}+$ $\widehat{\mathbf{v}}\left(t^{n-1}\right)$. If we apply $\left\|\widehat{\mathbf{E}}^{n-1}-\widehat{\mathbf{v}}\left(t^{n-1}\right)\right\|_{1} \leq C \tau^{\frac{1}{2}}$ again, then $A_{3,5}$ becomes

$A_{3,5}$

$$
\begin{aligned}
& =2 \tau \mathcal{N}\left(\mathbf{u}\left(t^{n-1}\right)-\widehat{\mathbf{E}}^{n-1}+\widehat{\mathbf{v}}\left(t^{n-1}\right), \delta \widehat{\mathbf{v}}\left(t^{n+1}\right), \delta \widehat{\mathbf{E}}^{n+1}\right) \\
& \leq C \tau\left(\left\|\mathbf{u}\left(t^{n-1}\right)\right\|_{2}\left\|\delta \widehat{\mathbf{v}}\left(t^{n+1}\right)\right\|_{0}+\left\|\widehat{\mathbf{E}}^{n-1}-\widehat{\mathbf{v}}\left(t^{n-1}\right)\right\|_{1}\left\|\delta \widehat{\mathbf{v}}\left(t^{n+1}\right)\right\|_{1}\right)\left\|\nabla \delta \widehat{\mathbf{E}}^{n+1}\right\|_{0} \\
& \leq \frac{\mu}{20} \tau\left\|\nabla \delta \widehat{\mathbf{E}}^{n+1}\right\|_{0}^{2}+\frac{C}{\mu} \tau\left(\left\|\delta \widehat{\mathbf{v}}\left(t^{n+1}\right)\right\|_{0}^{2}+\tau\left\|\nabla \delta \widehat{\mathbf{v}}\left(t^{n+1}\right)\right\|_{0}^{2}\right) .
\end{aligned}
$$

We impose $\left\|\mathbf{u}\left(t^{n}\right)-\mathbf{u}\left(t^{n-1}\right)\right\|_{1} \leq C \tau$ to have

$$
A_{3,6} \leq \frac{C}{\mu} \tau^{2} \int_{t^{n}}^{t^{n+1}}\left\|\mathbf{u}_{t}(t)\right\|_{1}^{2} d t+\frac{\mu}{20} \tau\left\|\nabla \delta \widehat{\mathbf{E}}^{n+1}\right\|_{0}^{2} .
$$

In conjunction with $\tau \sum_{n=1}^{N}\left\|\delta \mathbf{v}\left(t^{n+1}\right)\right\|_{0}^{2} \leq \int_{t^{0}}^{t^{N+1}}\left\|\mathbf{v}_{t}(t)\right\|_{0}^{2} d t$, collecting all these estimates and summing over $n$ from 1 to $N$ lead to

$$
\begin{aligned}
& \left\|\delta \mathbf{E}^{N+1}\right\|_{0}^{2}+\sum_{n=1}^{N}\left\|\delta \mathbf{E}^{n+1}-\delta \mathbf{E}^{n}\right\|_{0}^{2}+\frac{\mu}{2} \tau \sum_{n=1}^{N}\left\|\nabla \delta \widehat{\mathbf{E}}^{n+1}\right\|_{0}^{2}+\mu \tau\left\|\delta \varepsilon^{N+1}\right\|_{0}^{2} \\
& +2 \sum_{n=1}^{N}\left\|\nabla \delta \gamma^{n+1}\right\|_{0}^{2} \\
& \leq\left\|\delta \mathbf{E}^{1}\right\|_{0}^{2}+\mu \tau\left\|\delta \varepsilon^{1}\right\|_{0}^{2}+C \mu \tau^{2} \sum_{n=1}^{N}\left\|\delta \varepsilon^{n}\right\|_{0}^{2} \\
& +\frac{C}{\mu} \sum_{n=1}^{N}\left(\tau^{2}\left\|\nabla \delta \mathbf{E}^{n}\right\|_{0}^{2}+\tau\left\|\delta \mathbf{E}^{n}\right\|_{0}^{2}\right)+\frac{C}{\mu} \int_{t^{0}}^{t^{N+1}}\left(\left\|\mathbf{v}_{t t}\right\|_{0}^{2}+\left\|\nabla \mathbf{v}_{t}\right\|_{0}^{2}\left\|\nabla \widehat{\mathbf{v}}_{t}\right\|_{0}^{2}\right) d t \\
& +C(\mu) \tau^{2} \int_{t^{0}}^{t^{N+1}}\left(\left\|\mathbf{u}_{t}\right\|_{2}^{2}+\left\|\mathbf{u}_{t t}\right\|_{0}^{2}+\left\|\mathbf{u}_{t t t}\right\|_{-1}^{2}+\left\|\triangle \Phi_{t t}\right\|_{0}^{2}+\left\|\nabla \phi_{t t t}(t)\right\|_{0}^{2}\right) d t .
\end{aligned}
$$


We note that Lemma 5.2 leads $\left\|\delta \mathbf{E}^{1}\right\|_{0}^{2}+\mu \tau\left\|\delta \varepsilon^{1}\right\|_{0}^{2} \leq C \tau^{2}$ and that (4.4) derives $C \mu \tau^{2} \sum_{n=1}^{N}\left\|\nabla \delta \mathbf{E}^{n}\right\|_{0}^{2}$ cancellation with $\frac{\mu}{4} \tau \sum_{n=1}^{N}\left\|\nabla \delta \widehat{\mathbf{E}}^{n+1}\right\|_{0}^{2}$, if $\tau$ is small enough. The discrete Gronwall lemma allows us to remove $\tau \sum_{n=1}^{N}\left\|\delta \mathbf{E}^{n}\right\|_{0}^{2}+$ $C \mu \tau^{2} \sum_{n=1}^{N}\left\|\delta \varepsilon^{n}\right\|_{0}^{2}$. Finally, we apply (4.3) to obtain (5.5) and complete this proof.

\section{Error analysis for pressure}

The goal of this section is to estimate the pressure error for Algorithm 1. To do this, we first derive the following equation by inserting (1.9) into (1.7).

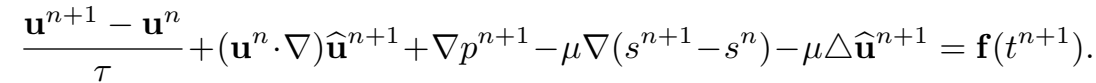

We now use Taylor theorem to derive

$$
\begin{aligned}
\frac{\mathbf{u}\left(t^{n+1}\right)-\mathbf{u}\left(t^{n}\right)}{\tau} & +\left(\mathbf{u}\left(t^{n+1}\right) \cdot \nabla\right) \widehat{\mathbf{u}}\left(t^{n+1}\right)+\nabla p\left(t^{n+1}\right) \\
& -\mu \triangle \mathbf{u}\left(t^{n+1}\right)=\mathbf{f}\left(t^{n+1}\right)+\mathbf{R}^{n+1},
\end{aligned}
$$

where $\mathbf{R}^{n+1}$ is defined at (3.7). If we use notations

$\widehat{\Xi}^{n+1}:=\mathbf{u}\left(t^{n+1}\right)-\widehat{\mathbf{u}}^{n+1}, \quad \Xi^{n+1}:=\mathbf{U}\left(t^{n+1}\right)-\mathbf{u}^{n+1}, \quad e^{n+1}:=p\left(t^{n+1}\right)-p^{n+1}$, then subtracting (6.1) from (6.2) yields

$$
\begin{gathered}
\frac{\Xi^{n+1}-\Xi^{n}}{\tau}+\nabla e^{n+1}-\mu \triangle \widehat{\Xi}^{n+1}=\mathbf{R}^{n+1}-\mu \nabla\left(s^{n+1}-s^{n}\right) \\
-\left(\mathbf{u}\left(t^{n+1}\right) \cdot \nabla\right) \mathbf{u}\left(t^{n+1}\right)+\left(\mathbf{u}^{n} \cdot \nabla\right) \widehat{\mathbf{u}}^{n+1} .
\end{gathered}
$$

This hinges on the error estimate for velocity in $\S 4$ and $\S 5$.

Lemma 6.1 (Rate of convergence for pressure). Suppose A1-6 hold. Then we have

$$
\left\|e^{n+1}\right\|_{0} \leq C \tau \text {. }
$$

Proof. We recall the existence of $\beta>0$ such that (inf-sup condition) [6]

$$
\beta\|q\|_{0} \leq \sup _{\mathbf{w} \in \mathbf{H}_{0}^{1}(\Omega)} \frac{\langle q, \nabla \cdot \mathbf{w}\rangle}{\|\nabla \mathbf{w}\|_{0}}, \quad \forall q \in L_{0}^{2}(\Omega) .
$$

Consequently, it suffices to estimate $\left\langle e^{n+1}, \nabla \cdot \mathbf{w}\right\rangle$ in terms of $\|\nabla \mathbf{w}\|_{0}$. Multiplying (6.3) by $\mathbf{w} \in \mathbf{H}_{0}^{1}(\Omega)$, we end up with

$$
\left\langle e^{n+1}, \nabla \cdot \mathbf{w}\right\rangle=\sum_{i=1}^{3} A_{i},
$$


where

$$
\begin{aligned}
& A_{1}=\left\langle\delta \Xi^{n+1}, \mathbf{w}\right\rangle+\mu\left\langle\nabla \widehat{\Xi}^{n+1}, \nabla \mathbf{w}\right\rangle-\left\langle\mathbf{R}^{n+1}, \mathbf{w}\right\rangle, \\
& A_{2}=-\mu\left\langle s^{n+1}-s^{n}, \nabla \cdot \mathbf{w}\right\rangle \\
& A_{3}=\mathcal{N}\left(\mathbf{u}\left(t^{n+1}\right), \mathbf{u}\left(t^{n+1}\right), \mathbf{w}\right)-\mathcal{N}\left(\mathbf{u}^{n}, \widehat{\mathbf{u}}^{n+1}, \mathbf{w}\right) .
\end{aligned}
$$

We now proceed to estimate each term $A_{1}$ to $A_{3}$ separately. In view of Hölder inequality, $A_{1}$ becomes

$$
A_{1} \leq C\left(\left\|\delta \Xi^{n+1}\right\|_{0}+\mu\left\|\nabla \widehat{\Xi}^{n+1}\right\|_{0}+\tau\left\|\mathbf{u}_{t t}\right\|_{-1}\right)\|\nabla \mathbf{w}\|_{0} .
$$

On other hand, we have

$$
\begin{aligned}
A_{2} & =\mu\left\langle\nabla \cdot \widehat{\mathbf{u}}^{n+1}, \nabla \cdot \mathbf{w}\right\rangle \\
& =-\mu\left\langle\nabla \cdot \widehat{\Xi}^{n+1}, \nabla \cdot \mathbf{w}\right\rangle \leq C\left\|\nabla \widehat{\Xi}^{n+1}\right\|_{0}\|\nabla \mathbf{w}\|_{0} .
\end{aligned}
$$

The remaining term $A_{3}$ can be further split as follow:

$$
A_{3}=\mathcal{N}\left(\mathbf{u}\left(t^{n+1}\right)-\mathbf{u}\left(t^{n}\right)+\Xi^{n}, \mathbf{u}\left(t^{n+1}\right), \mathbf{w}\right)+\mathcal{N}\left(\mathbf{u}\left(t^{n}\right)-\Xi^{n}, \widehat{\Xi}^{n+1}, \mathbf{w}\right)
$$

and it can be bounded by

$$
\begin{aligned}
A_{3} \leq & C\left(\left\|\mathbf{u}\left(t^{n+1}\right)-\mathbf{u}\left(t^{n}\right)\right\|_{0}+\left\|\Xi^{n}\right\|_{0}\right)\left\|\mathbf{u}\left(t^{n+1}\right)\right\|_{2}\|\nabla \mathbf{w}\|_{0} \\
& +C\left(\left\|\mathbf{u}\left(t^{n}\right)\right\|_{1}+\left\|\Xi^{n}\right\|_{1}\right)\left\|\widehat{\Xi}^{n+1}\right\|_{1}\|\nabla \mathbf{w}\|_{0} .
\end{aligned}
$$

Inserting the estimates for $A_{1}$ to $A_{3}$ back into (6.6), and employing (6.5), we obtain

$$
\begin{aligned}
\beta\left\|e^{n+1}\right\|_{0} \leq & C\left(\left\|\delta \Xi^{n+1}\right\|_{0}+\mu\left\|\nabla \widehat{\Xi}^{n+1}\right\|_{0}+\tau\left\|\mathbf{u}_{t t}\right\|_{-1}\right) \\
& +C\left(\left\|\mathbf{u}\left(t^{n+1}\right)-\mathbf{u}\left(t^{n}\right)\right\|_{0}+\left\|\Xi^{n}\right\|_{0}\right) .
\end{aligned}
$$

Finally, combining the results of Lemmas 4.1 and 5.3, we obtain (6.4) and complete the proof.

\section{References}

[1] D. L. Brown, R. Cortez, and M. L. Minion, Accurate projection methods for the incompressible Navier-Stokes equations, J. Comput. Phys. 168 (2001), no. 2, 464-499.

[2] A. J. Chorin, Numerical solution of the Navier-Stokes equations, Math. Comp. 22 (1968), 745-762.

[3] P. Constantin and C. Foias, Navier-Stokes Equations, The University of Chicago Press, 1988.

[4] M. Dauge, Stationary Stokes and Navier-Stokes systems on two- or three-dimensional domains with corners. I. Linearized equations, SIAM J. Math. Anal. 20 (1989), no. 1, 74-97.

[5] Weinan E. and J.-G. Liu, Gauge method for viscous incompressible flows, Comm. Math. Sci. 1 (2003), 317-332.

[6] V. Girault and P. A. Raviart, Finite Element Methods for Navier-Stokes Equations, Springer Series in Computational Mathematics, 5. Springer-Verlag, Berlin, 1986.

[7] J. G. Heywood and R. Rannacher, Finite element approximation of the nonstationary Navier-Stokes problem. I. Regularity of solutions and second-order error estimates for spatial discretization, SIAM J. Numer. Anal. 19 (1982), no. 2, 275-311. 
[8] R. B. Kellogg and J. E. Osborn, A regularity result for the Stokes problem in a convex polygon, J. Functional Analysis 21 (1976), no. 4, 397-431.

[9] R. H. Nochetto and J.-H. Pyo, Optimal relaxation parameter for the Uzawa method, Numer. Math. 98 (2004), no. 4, 695-702.

[10] _ Error estimates for semi-discrete gauge methods for the Navier-Stokes equations, Math. Comp. 74 (2005), no. 250, 521-542.

[11] _ The gauge-Uzawa finite element method. I. The Navier-Stokes equations, SIAM J. Numer. Anal. 43 (2005), no. 3, 1043-1068.

[12] _ The gauge-Uzawa finite element method. II. The Boussinesq equations, Math. Models Methods Appl. Sci. 16 (2006), no. 10, 1599-1626.

[13] A. Prohl, Projection and Quasi-Compressibility Methods for Solving the Incompressible Navier-Stokes Equations, Advances in Numerical Mathematics. B. G. Teubner, Stuttgart, 1997.

[14] J.-H. Pyo, The gauge-Uzawa and related projection finite element methods for the evolution Navier-Stokes equations, Ph.D. dissertation, University of Maryland, 2002.

[15] J.-H. Pyo and J. Shen, Normal mode analysis of second-order projection methods for incompressible flows, Discrete Contin. Dyn. Syst. Ser. B 5 (2005), no. 3, 817-840.

[16] _ Gauge-Uzawa methods for incompressible flows with variable density, J. Comput. Phys. 221 (2007), no. 1, 181-197.

[17] R. Témam, Sur l'approximation de la solution des equations de Navier-Stokes par la methode des pas fractionnaires. II, Arch. Rational Mech. Anal. 33 (1969), 377-385.

[18] C. Wang and J.-G. Liu, Convergence of gauge method for incompressible flow, Math. Comp. 69 (2000), no. 232, 1385-1407.

Department of Mathematics

KANGWON NATIONAL UNIVERSITY

Chuncheon 200-701, KoreA

E-mail address: jhpyo@kangwon.ac.kr 